\title{
Wir versorgen wirklich jeden!
}

\author{
Flüchtlinge, Obdachlose, Zugewanderte, durchaus auch Selbstständige - viele Menschen in \\ Deutschland haben keine Krankenversicherung. Die Initiative Open.med behandelt sie trotzdem.
}

\begin{abstract}
_Open.med - der Name ist Programm: Seit November betreibt der Verein „Medizin hilft“ in Kooperation mit „Ärzte der Welt“ einen Sprechstundenraum in der Zehlendorfer Kirchstraße in Berlin. Jeden Donnerstagnachmittag steht die Tür für alle Menschen offen, die Beratung oder medizinische Hilfe benötigen.
\end{abstract} Schon bald sollen die Sprechstundenzeiten ausgedehnt werden; auch der Umzug in größere Räumlichkeiten am Teltower Damm ist bereits geplant.

Open.med ist das zweite Projekt des Vereins „Medizin hilft“. Er formierte sich vor zwei Jahren, als die Flüchtlingskrise in Berlin zu immer schlimmeren Zuständen führte. Damals, kurz vor Weihnachten 2014, schaute die Sportmedizinerin Dr. Pia Skarabis-Querfeld in einer Notunterkunft vorbei. Eigentlich wollte sie nur Sachspenden vorbeibringen - doch als sie sah, dass überhaupt keine medizinische Versorgung existierte, verbrachten ihr Mann und sie die Festtage damit, die Flüchtlinge zu behandeln.

Innerhalb kurzer Zeit gelang es Skarabis-Querfeld mit Unterstützung der evangelischen Gemeinde, ein Netzwerk von 120 Ehrenamtlichen aufzubauen, das sich bald um vier bis fünf Flücht- lingseinrichtungen kümmerte. Im April 2016 wurde schließlich der Verein „Medizin hilft" gegründet. Er finanziert sich aus Spendengeldern, vornehmlich von elf Rotary-Clubs aus Deutschland, Österreich, Frankreich und den USA.

\section{Selbstständige sind oft betroffen}

Mit Open.med will „Medizin hilft“ nun eine zentrale Anlaufstelle für alle Menschen schaffen, die - warum auch immer - keine Krankenversicherung haben. „Wir wollen alle Menschen versorgen, die keinen Zugang zum Gesundheitssystem haben", sagt die Vereinsvorsitzende Skarabis-Querfeld.

Das umfasst nicht nur Flüchtlinge, die etwa ihren Behandlungsschein verloren haben, sondern auch Obdachlose, EU-Bürger ohne Versicherungsschutz oder mittellose Selbstständige über 55 Jahren, für welche die Rückkehr von der privaten in die gesetzliche Krankenver- sicherung ausgeschlossen ist. In München, wo „Ärzte der Welt" schon seit zehn Jahren eine Anlaufstation betreibt, sind diese Selbstständigen laut der Berliner Projektreferentin Burcu Güvenc die größte Patientengruppe. Die zweitgrößte Gruppe sind die EU-Bürger, die keinen Versicherungsschutz haben.

\section{0 Ärzte helfen}

In Berlin kann das Projekt bereits auf ein Netzwerk von rund 100 Ärzten zurückgreifen, berichtet Barbara Grube. Die Hausärztin ist ärztliche Leiterin bei Open.med. „Wir brauchen aber auch noch mehr Fachärzte“, sagt sie. Denn das Ziel ist es, auch Sprechstunden speziell für Frauen, Kinder und chronisch Kranke anzubieten. Zudem, so die stellvertretende Vorsitzende Dr. Laura Hatzler, solle die Qualität des Angebots so hoch sein wie in den Praxen.

Derzeit arbeitet „Medizin hilft“ an einem weiteren Konzept: Auf Informationsveranstaltungen soll Flüchtlingen und anderen Interessierten das deutsche Gesundheitssystem erklärt werden. Auch will man die Ärzte in der ambulanten Versorgung besser über die gesetzlichen Behandlungsmöglichkeiten aufklären - und ihnen die oft vorhandene $\mathrm{Zu-}$ rückhaltung bei der Versorgung von Flüchtlingen nehmen.

Julia Frisch 